\title{
Toxic $Y$ chromosome: increased repeat expression and age-associated heterochromatin loss in male Drosophila with a young $Y$ chromosome
}

\author{
Alison H. Nguyen \& Doris Bachtrog \\ Department of Integrative Biology, University of California Berkeley, Berkeley, CA 94720, USA
}

Sex-specific differences in lifespan are prevalent across the tree of life and influenced by heteromorphic sex chromosomes. In species with XY sex chromosomes, females often outlive males. Males and females can differ in their overall repeat content due to the repetitive $Y$ chromosome, and repeats on the $Y$ might lower survival of the heterogametic sex (toxic $Y$ effect). Here, we take advantage of the well-assembled young $Y$ chromosome of Drosophila miranda to study the sex-specific dynamics of chromatin structure and repeat expression during aging in male and female flies. Male $D$. miranda have about twice as much repetitive DNA compared to females, and live shorter than females. Heterochromatin is crucial for silencing of repetitive elements, yet old $D$. miranda flies lose H3K9me3 modifications in their pericentromere, with heterochromatin loss being more severe during aging in males than females. Satellite DNA becomes de-repressed more rapidly in old vs. young male flies relative to females. In contrast to what is observed in $D$. melanogaster, we find that transposable elements (TEs) are expressed at higher levels in male $D$. miranda throughout their life. We show that epigenetic silencing via heterochromatin formation is ineffective on the large TErich neo-Y chromosome, resulting in up-regulation of Y-linked TEs already in young males. This is consistent with an interaction between the age of the $Y$ chromosome and the genomic effects of aging. Our data support growing evidence that "toxic $Y$ chromosomes" can diminish male fitness and a reduction in heterochromatin can contribute to sex-specific aging.

\section{Introduction}

Males and females differ in many life history traits, and sexual dimorphism in fitness-related traits is often driven by natural selection [1]. However, some traits could vary between the sexes in a nonadaptive manner. For example, males and females often differ in their lifespan [2], and the chromosomal sex determination system has been shown to influence sex-specific longevity. In particular, several studies have suggested that the sex with the heteromorphic sex chromosomes (males in XY species; females in ZW species) has a shorter lifespan on average [3,4]. The aging process is associated with an overall loss of heterochromatin in many species $[5,6]$, and differences in the heterochromatin content between sexes could in principle contribute to sex-specific mortality (the toxic $Y$ effect; [7]). In species with heteromorphic sex chromosomes, the amount of repetitive DNA and thus heterochromatin can vary dramatically between males and females, due to the presence of a large, repetitive $\mathrm{Y}$ (or $\mathrm{W}$ ) chromosome in the heterogametic sex. Heterochromatin loss can result in derepression and mobilization of silenced transposable elements (TEs) [8-11], and might disproportionally affect the sex with the higher heterochromatin content.

We previously showed that heterochromatin loss differs between the sexes in D. melanogaster [12]. TEs are up-regulated in old male flies, and Y-linked repeats are especially prone to become de-repressed during aging in $D$. melanogaster. Genetic manipulations of sex chromosome karyotypes suggested that the $\mathrm{Y}$ chromosome directly contributes to sex-specific aging in flies: females containing a $\mathrm{Y}$ chromosome 
(XXY females) lived shorter than wildtype XX females, males lacking a $Y$ (XO males) outlived wildtype males, and males containing two $Y$ chromosomes (XYY males) showed a drastically reduced lifespan compared to $X Y$ males [12]. However, repeat-rich $Y$ chromosomes are typically poorly assembled (only $14.6 \mathrm{Mb}$ of the roughly $40-\mathrm{Mb}$ large $\mathrm{Y}$ chromosome of $D$. melanogaster is present in the latest assembly; [13]), making it impossible to directly observe chromatin changes on the heterochromatic $Y$ chromosome and link them to expression changes of repeats using genomic approaches.

A

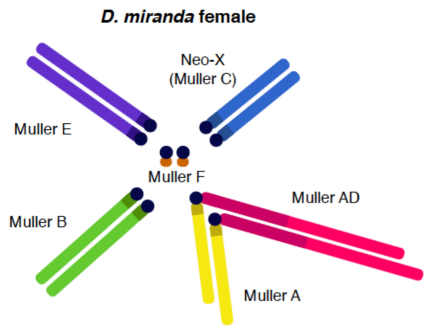

C

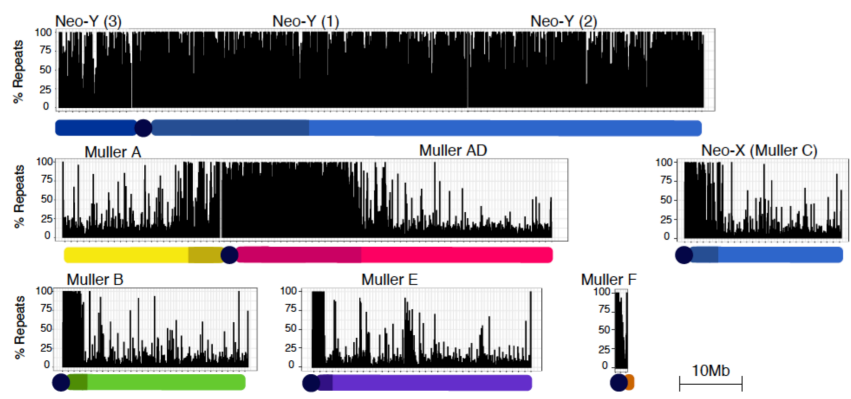

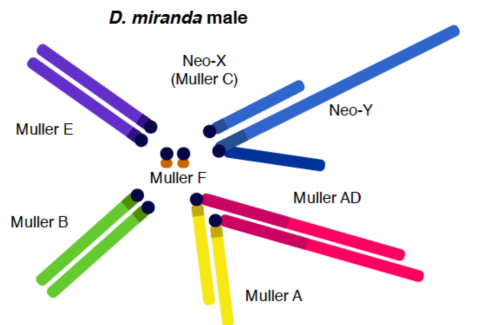

D
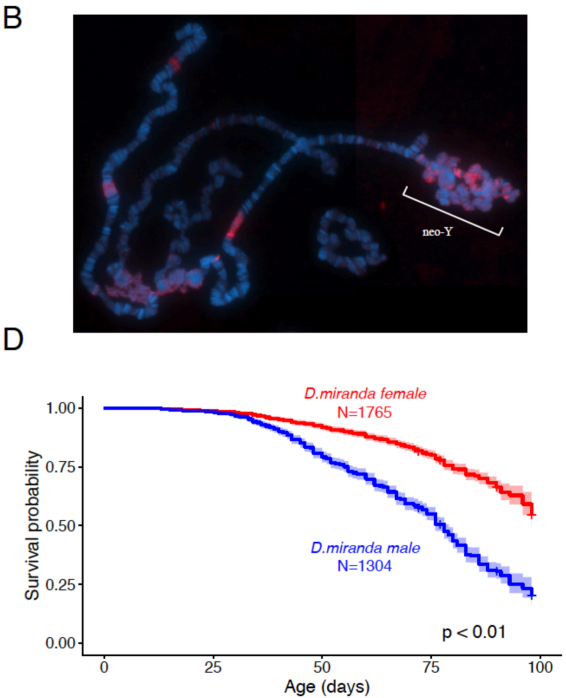

Figure 1. Drosophila miranda karyotype and lifespan. A. Karyotype of $D$. miranda males and females. Chromosome arms (Muller elements) are color-coded. B. Polytene chromosome squash of D. miranda male stained with HP1 (magenta) marking heterochromatin. C. Genome-wide repetitive content per chromosome. Shown is repeat content (\% repeat masked bp) in $50 \mathrm{~kb}$ windows. D. Kaplan-Meier survivorship curves [67] for MSH22 males (blue) and females (red), with the shaded region indicating the upper and lower $95 \%$ confidence interval calculated from the Kaplan-Meier curves. The number of flies counted for each sex $(n)$ to obtain the survivorship curves is indicated.

Here, we test for an association between heteromorphic sex chromosomes and sex-specific heterochromatin formation and silencing of repetitive DNA in a species with a well-assembled $Y$ chromosome [14]. We assay chromatin and gene expression profiles in young and aged males and females of $D$. miranda (Figure 1A), a model system for studying the molecular basis of sex chromosome differentiation [14-19]. D. miranda has a recently evolved neo-sex chromosome that was formed only 1.5MY ago from an ordinary autosome that fused to the ancestral $Y[20]$. The neo- $X$ and neo- $Y$ are in the process of differentiating into heteromorphic sex chromosomes, and the neo- $Y$ has dramatically increased in size mainly through an accumulation of TEs $[14,15,17,21]$. Specifically, a high-quality genome assembly that includes large fractions of heterochromatin contained $>90 \mathrm{Mb}$ of neo-Y-linked sequence (compared to $\sim 25 \mathrm{Mb}$ of neo-X linked sequence), and $65 \mathrm{Mb}$ are derived from repetitive elements (compared to $5 \mathrm{Mb}$ on the neo-X) [14]. TEs are uniformly enriched along the neo-Y chromosome and a main contributor to its dramatically increased genome size [14, 15, 17, 21]. For example, the most abundant repeat on the neo- $Y$ is the ISY element, a helitron transposon that is inserted about 22,000 times on the neo-Y/Y chromosome and amounts to more than $16 \mathrm{Mb}$ of DNA (i.e., $16 \%$ of neo-Y sequence), while its former homolog, the neo-X chromosome only harbors about 1,500 copies (3\% of the neo-X; less than $1 \mathrm{Mb}$; [14]). The second most common repeat class that has amplified on the neo-Y are gypsy TEs; roughly 14,300 insertions were found on the neo-Y (15\% of neo-Y sequence, 
$15 \mathrm{Mb}$ ) and less than $1 \mathrm{Mb}$ on the neo-X (about 800 insertions, i.e., 3\% of its sequence; [14]). Thus, a huge number of TEs has accumulated on the neo-Y chromosome since its origination $1.5 \mathrm{MY}$ ago. Yet this chromosome still contains thousands of protein-coding genes that are embedded within these islands of TEs [14], and many of the neo-Y linked protein-coding genes are still actively transcribed [18, 22]. Thus, unlike the $Y$ chromosome of $D$. melanogaster, the neo- $Y$ of $D$. miranda contains thousands of transcribed, euchromatic genes, which are intermingled with repetitive heterochromatin.

We use chromatin and transcription profiling to show that heterochromatin formation is comprised on the repeat-rich neo-Y of $D$. miranda already in young males, resulting in global up-regulation of TEs in males. Age-associated heterochromatin loss is more pronounced in male flies compared to females, which also live shorter, and accompanied by de-repression of satellite repeats in males. Our data provide empirical support that toxic $Y$ chromosomes can diminish male fitness, and contribute to sexspecific aging in species with heteromorphic sex chromosomes.

\section{Results}

Drosophila miranda males have more repeats and live shorter.

We chose the standard lab strain MSH22 of $D$. miranda, a fly that has served as a model for $Y$ chromosome evolution (Figure 1A) $[14,18,22]$ to investigate how the 'toxic' $Y$ chromosome may influence sex-specific behaviors of repetitive sequences, such as TE expression, the dynamics of heterochromatin during the lifespan of a fly, and sex-specific aging. In D. miranda, an autosome fused to the ancestral sex chromosome about 1.5MY ago [20], creating a neo-Y chromosome which is at an intermediate stage of degeneration. The neo-Y still contains many of its ancestral genes, but has expanded in size by about 3-fold, mostly due to the accumulation of TEs $[14,17]$. This increase in repeat content is accompanied by a change in its chromatin structure with many regions along the neo-Y becoming heterochromatic (Figure 1B) [23]. D. miranda has a high-quality genome assembly that comprise large stretches of repetitive heterochromatin, including major parts of the pericentromeres and the $Y$ chromosome [14] (Figure 1C). In particular, D. miranda males have a enormous, $92 \mathrm{Mb}$ large recently formed and highly repetitive and heterochromatic neo-Y chromosome [14], while the pericentromeric heterochromatin on the $X /$ neo-X only amounts to $24 \mathrm{Mb}$ (Table 1) [14, 24]. Thus, males contain significantly more repetitive DNA than females, and flow cytometry estimates agree with our assembled genome sizes (Table 1).

Table 1 Assembled length for each chromosome, size of chromosome arms and pericentromere, and repeatmasked bases (in Mb). The total genome size inferred from flow cytometry is given in brackets.

\begin{tabular}{lcccc}
\hline & $\begin{array}{c}\text { Total Assembly } \\
(\mathrm{Mb})\end{array}$ & $\begin{array}{c}\text { Euchromatic Arms } \\
(\mathrm{Mb})\end{array}$ & $\begin{array}{c}\text { Pericentric } \\
\text { Heterochromatin }\end{array}$ & $\begin{array}{c}\text { Repeatmasked } \\
(\mathrm{Mb})\end{array}$ \\
\hline $\mathrm{X}$ chr (A/D) & 77.6 & 57.1 & 20.5 & 28.9 \\
neo-X (C) & 25.3 & 21.9 & 3.4 & 6.1 \\
Y/neo-Y chr & 92.1 & $\mathrm{n} / \mathrm{a}$ & $\mathrm{n} / \mathrm{a}$ & 76.8 \\
chr4 (B) & 32.5 & 29.1 & 3.4 & 6.6 \\
chr2 (E) & 35.3 & 33.3 & 2 & 6.2 \\
dot & 2.4 & 0 & 2.4 & 1.3 \\
total & 173.1 & 141.4 & 31.7 & 49.1 \\
male (2n) & $335.4(354)$ & 203.8 & 131.6 & 140.0 \\
female (2n) & $346.3(351.2)$ & 282.9 & 63.4 & 98.2 \\
\hline
\end{tabular}


In most Drosophila species male flies live significantly shorter than female flies $[25,26]$. We determined longevity for males and females for the reference lab strain from D. miranda (MSH22) at $18^{\circ} \mathrm{C}$ (from 3 biological replicates, see Methods). Median survivorship for males is 78 days, and $>98$ days for females (Figure 1D). Thus, lifespan assays confirm that males live significantly shorter than females, consistent with multiple studies on sex-specific lifespan in Drosophila $[25,26]$. Note however that other factors besides sex (such as male-male aggression) may contribute to differences in longevity between males and females [27]. Longevity patterns are thus consistent with the notion that the repeat-rich $Y$ chromosome reduces survivorship in the heterogametic sex $[3,4]$.

\section{Heterochromatin loss is more pronounced in males.}

We collected replicate ChIP-seq data (4 replica for each sex and age) for a histone modification typical of repressive heterochromatin (H3K9me3) from young (9-11 days) and old (80-90-day) D. miranda male and female brains (MSH22) (Table S1). We spiked-in a fixed amount of chromatin from D. melanogaster to each $D$. miranda chromatin sample prior to ChIP and sequencing, to compare the genomic distribution of chromatin marks across samples using a 'spike in' normalization method $[12,28]$. This species pair is sufficiently diverged from each other that there is very little ambiguity in the assignment of reads to the correct species, and this strategy allows us to compare levels of H3K9me3 enrichment across sexes and time.

Figure 2 shows the genomic distribution of the repressive histone modification H3K9me3 for young and old male and female flies. As expected, heterochromatin is enriched at repetitive regions, including pericentromeres, the small dot chromosome and the repeat-rich $Y$ chromosome (Figure 2A). Overall heterochromatin enrichment is similar for repetitive regions at the $X$ and autosomes in young males and females (Figure 2B). Consistent with heterochromatin being lost during aging, both males and females show a reduction in $\mathrm{H} 3 \mathrm{~K} 9 \mathrm{me} 3$ enrichment at their pericentromeres in old compared to young flies (Figure 2, 3). Strikingly, heterochromatin loss is more pronounced in old male flies than in old females (Figure 2A, B). This finding is reproducible across individual replicates (Figures S1-S6), and robust with regards to normalization strategy (Figure S1) and using only uniquely mapping reads (Figure S3). $D$. miranda males show significantly more regions that lose H3K9me3 signal (1.5-fold or more in 5-kb windows) during aging compared to females (2936 vs. 316, $p<0.00001$; Fisher's exact test; Figure S7). Almost all regions ( $78 \%$ of windows) that lose heterochromatin in males are located within the pericentromeres of the autosome/X (1963 windows) and the neo-Y (328 windows) (Figure S7, S8). In females, $25.6 \%$ of windows (81) losing heterochromatin occur within the pericentromeres. Many fewer regions gain H3K9me3 signal (1.5-fold or more) during aging (947 in males and 155 in females, $\mathrm{p}<$ 0.00001 , Fisher's exact test; Figure S7). Almost all regions that gain H3K9me3 signal are found along the chromosome arms (Figure S7), consistent with the idea that some heterochromatin becomes redistributed in old flies [29]. Faster heterochromatin loss in old males is in agreement with recent findings in $D$. melanogaster [12]. These data suggest that increased deterioration of heterochromatin during aging may be a general feature of the sex with more repetitive DNA.

\section{Heterochromatin content and loss on the neo-Y chromosome.}

The neo- $Y$ is highly repetitive (Figure 1, Table 1 ) and enriched for heterochromatin (Figure 2), yet to a lesser extent than pericentromeric regions (Figure 2A, B). The neo-Y harbors several thousand proteincoding genes embedded in highly repeat-rich regions [14, 22], and overall repeat content on the neo- $Y$ is slightly less than of pericentromeres on the $X$ or autosomes ( $72 \%$ of bp are repeat-masked on the neo- $Y$ chromosome, compared to $82-89 \%$ of the pericentromeres on $\mathrm{X}$ and autosomes). 
A

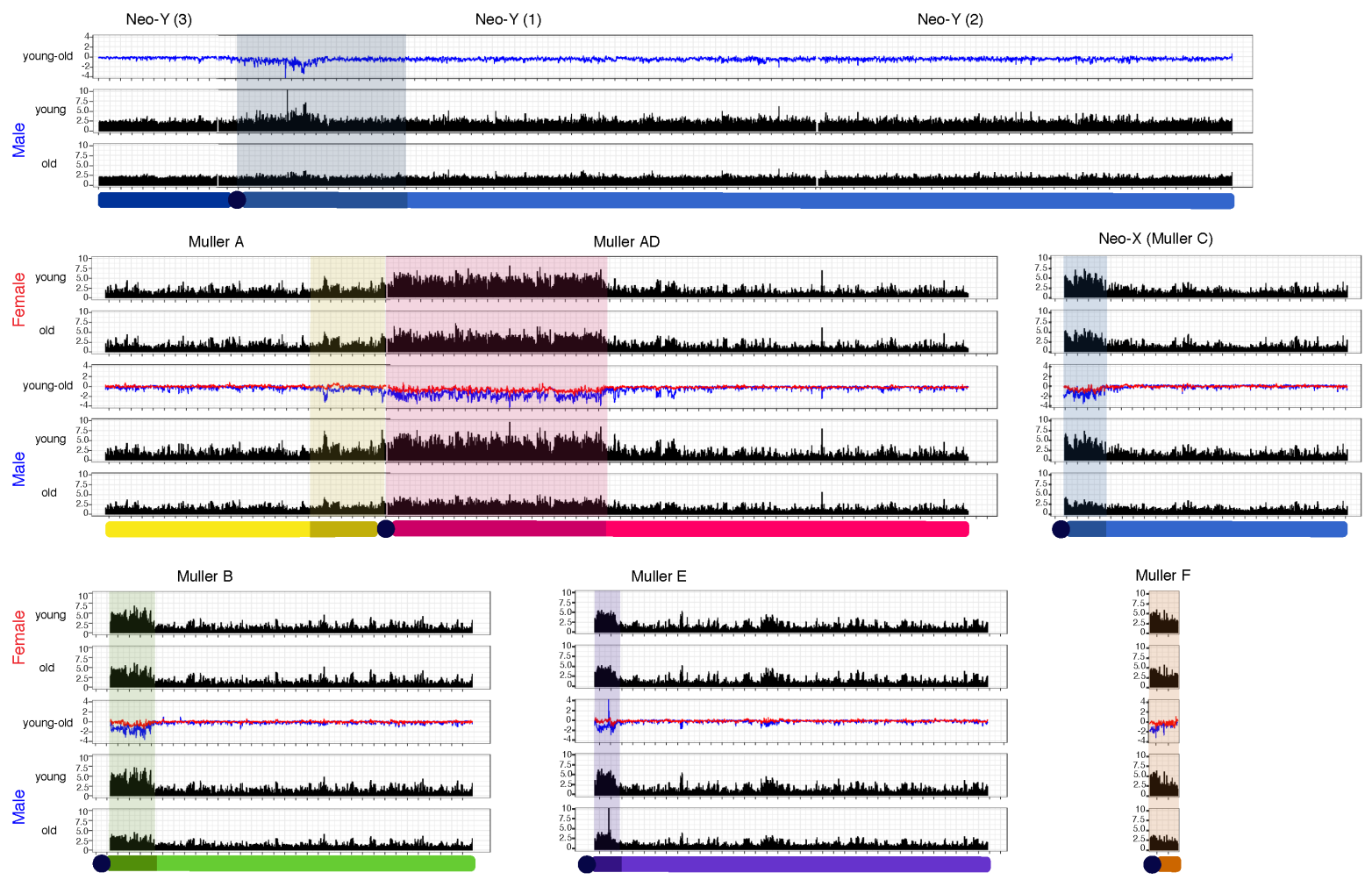

B

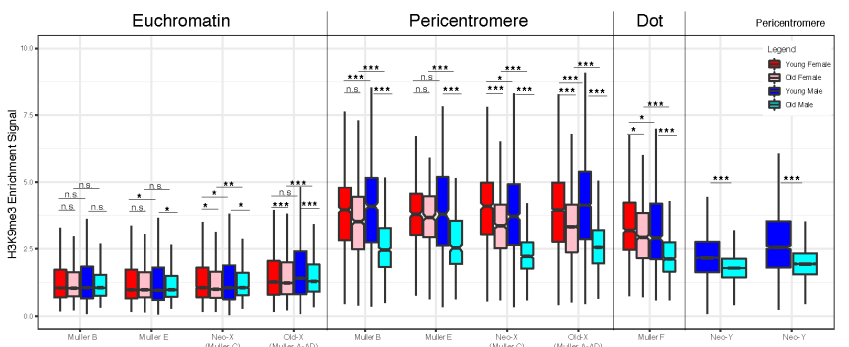

Figure 2. Heterochromatin loss and aging. A. Genome-wide enrichment of H3K9me3 for young and old D. miranda males and females along the different chromosome arms. Values represent the mean of 4 biological replicates for Young/Old Females and Young Males and the mean of 3 biological replicates for Old Males in $25 \mathrm{~kb}$ windows. Subtraction plots show the absolute difference in signal of $25 \mathrm{~kb}$ windows between young and aged flies along the chromosome arms, with females in red and males in blue. One tick mark is equal to $1 \mathrm{Mb}$ on the $\mathrm{x}$-axis. B. Boxplot showing ChIP enrichment differences between biological samples at euchromatin and pericentromeres. Enrichment resolution is in $5 \mathrm{~kb}$ windows. Significance values calculated $(* p<0.05, * * p<1 e-6, * * * p<1 e-12$, Wilcoxon test) for males (blue, cyan) and females (red, pink), with pericentromere boundaries defined by [24].

To evaluate if lower repeat content on the neo- $Y$ can account for lower levels of heterochromatin, we compared H3K9me3 enrichment versus repeat content for 5-kb windows across chromosomes (Figure S9). As expected, genomic regions with a higher repeat density show more heterochromatin, and absolute levels of H3K9me3 enrichment are very similar between the $\mathrm{X}$ and autosomes, and young males and females. However, for repeat-rich windows ( $>70 \%$ repeat masked), the neo- $Y$ chromosome shows considerably lower levels of $\mathrm{H} 3 \mathrm{~K} 9 \mathrm{me} 3$ enrichment than other chromosomes, even in young males 
(Figure S9). In particular, average H3K9me3 enrichment in highly repetitive windows ( $>70 \%$ of bases repeatmasked) is about 30\% lower on the neo-Y compared to X-linked or autosomal regions in young males, and $24 \%$ lower in old males. The same conclusion holds if the top repeat-rich regions $(>99.5 \%$ repeat masked) are considered (Figure S9). Thus, this suggests that repetitive DNA on the neo-Y shows less heterochromatin-induced epigenetic silencing than other repeat-rich regions of the genome.

Multiple factors could contribute to lower levels of H3K9me3 enrichment on the neo-Y. The neo-Y chromosome still contains many unique regions with transcribed protein-coding genes $[14,18,22]$ and thus encompasses a patchwork of heterochromatic repeats interspersed with unique and genetically active euchromatic regions. In Drosophila, the boundary between heterochromatin and euchromatin is not fixed but determined by the local balance between factors that promote either heterochromatin or euchromatin [30]. Active transcription could limit spreading of heterochromatin on the neo-Y and reduce overall H3K9me3 enrichment [31]. Indeed, the gene density on the neo-Y is substantially higher than in the pericentromeres. The $D$. miranda annotation contains 255 genes located in the pericentromeres (that is, roughly 9 genes/Mb) and 5625 genes on the $\mathrm{Y} /$ neo- $\mathrm{Y}$ (61 genes/Mb) [14]. Most genes are transcribed in adult brain samples (246 of all the pericentromeric genes and 4428 of the neo-Y genes; see below), consistent with the idea that active transcription of neo- $Y$ genes could impede heterochromatin formation on this chromosome.

Heterochromatin on the neo-Y is evolutionarily young. The neo-Y was an ordinary autosome until $1.5 \mathrm{MY}$ ago and only acquired its high repeat content and heterochromatin structure after it became fused to the ancestral $Y[15,21]$. The repeats present on the neo-Y should therefore encompass mostly recently active TEs that may initiate less heterochromatin [32], and its overall repeat structure may differ from pericentromeres. Indeed, the relative abundance of different TE families differs between the neo- $Y$ chromosome and the rest of the genome (Figure S10; [14]); we find the neo-Y contains more LTR transposons and helitrons but less simple repeats in comparison to other chromosomes (Figure S10).

While overall TE composition differs between the neo-Y and other chromosomes, transposons belonging to the same family may also show lower levels of H3K9me3 enrichment if present on the neo-Y. As expected, H3K9me3 profiles at TE families show that there is a general enrichment of this repressive mark in both male and female flies (Figure 3A). To test if neo-Y linked TEs show less H3K9me3 enrichment, we calculated average H3K9me3 enrichment for each TE family based on genome-wide mapping of H3K9me3 separately for copies located on the neo-Y versus other genomic locations. Indeed, copies of TEs inserted onto the neo-Y typically show lower levels of H3K9me3 enrichment than copies on other chromosomes. Global H3K9me3 enrichment is about 30\% lower for neo-Y TEs compared to their $X$ - and autosomal homologs in young flies (and 27\% lower in old flies), and 92/16 show significantly less/more $\mathrm{H} 3 \mathrm{~K} 9 \mathrm{me} 3$ if located on the neo-Y relative to copies on the X/autosome in young males (and 25/6 in old ones; Figure 3B). Thus, this suggests that most TEs experience less epigenetic silencing on the neo- $Y$ relative to other regions of the genome.

Like other repeat-rich regions, overall levels of heterochromatin on the neo-Y decrease; however, the absolute amount of $\mathrm{H} 3 \mathrm{~K} 9 \mathrm{me} 3$ loss appears less on the $\mathrm{Y}$ in old males, compared to pericentric regions, or the dot chromosome (Figure 2, Figure S11). Overall, our results show that male $D$. miranda lose heterochromatin marks more rapidly than female, consistent with our previous findings in $D$. melanogaster [12]. However, we also find that the neo-Y shows considerably lower levels of heterochromatin than what we might expect given its repeat content. Indeed, levels of H3K9me3 enrichment on the neo- $Y$ in young males resemble reduced levels of heterochromatin at $X$-linked and autosomal pericentromeres in old males. 

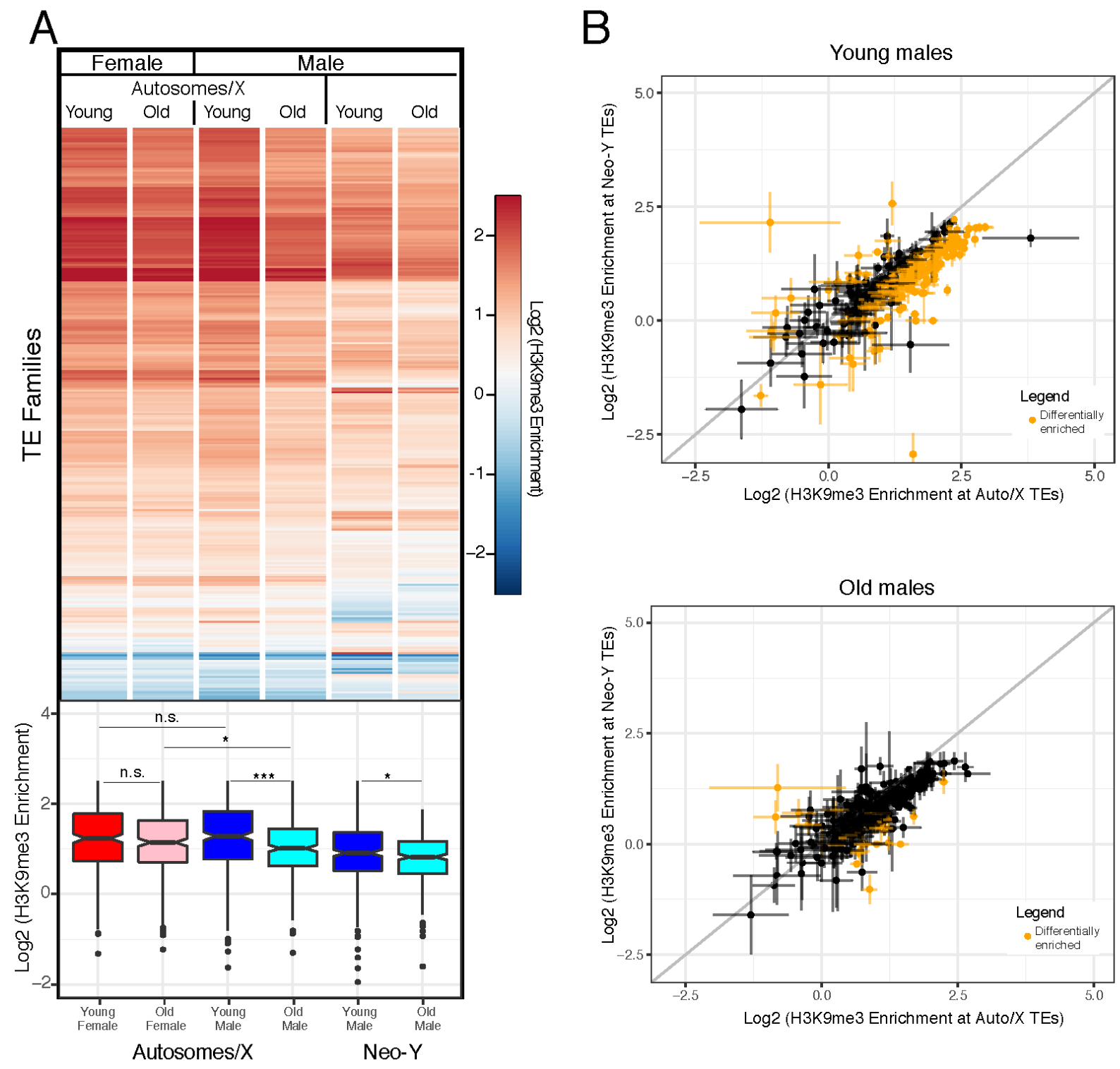

Figure 3. Heterochromatin enrichment at TEs across sex, time and chromosomes. Shown is enrichment of H3K9me 3 at different repeat families for young and old females and males. A. Log2 H3K9me3 enrichment of all transposable elements (from [61]) in young (5-9 day) and old (80-98 day) male and female MSH22, averaged across 4 replicates for Young/Old Females and Young Males and across 3 replicates for Old Males. H3K9me3 enrichment in males is separated by autosomes/ $X$ and neo-Y. Boxplots below with significance values calculated using the Wilcoxon test $\left({ }^{*} p<0.05,{ }^{* *} p<0.01\right.$, $* * * \mathrm{p}<1 \mathrm{e}-5$ ). B. Log2(H3K9me3 enrichment) differences between TEs found on the Autosomes/ $\mathrm{X}$ and the neo-Y for young (top) and old (bottom) males. Data represents averages of 4 replicates for Young/Old Females and Young Males and of 3 replicates for Old Males. The bars denote standard error. Data highlighted in yellow are TEs that show significant differences in H3K9me3 enrichment on autosomes/X and neo-Y ( $p<0.05$, two-tailed Student's t-test).

\section{Repeat de-repression in young male flies}

Heterochromatin silences repetitive DNA [31], and reduced levels of H3K9me3 along the large repeatrich neo-Y chromosome could have profound consequences on TE silencing in male $D$. miranda even in young flies. To study TE expression changes between sexes and during aging, we gathered replicated 
stranded RNA-seq data from young and old D. miranda (3 biological replicates; Table S2). Indeed, comparison of TE expression between young and old male and female $D$. miranda reveals that repeat expression is strikingly increased in male flies compared to females, irrespective of age (Figure 4A,B). In particular, 135 TE families are significantly upregulated, while 1 is expressed at lower levels in male $D$. miranda compared to females already in 9-day old flies, and the fraction of transcripts derived from TEs is more than twice as high in males compared to females (the fraction of repetitive reads in all RNA-seq reads is on average $8.0 \%$ at young males and $3.7 \%$ at young females, Table S3).

A

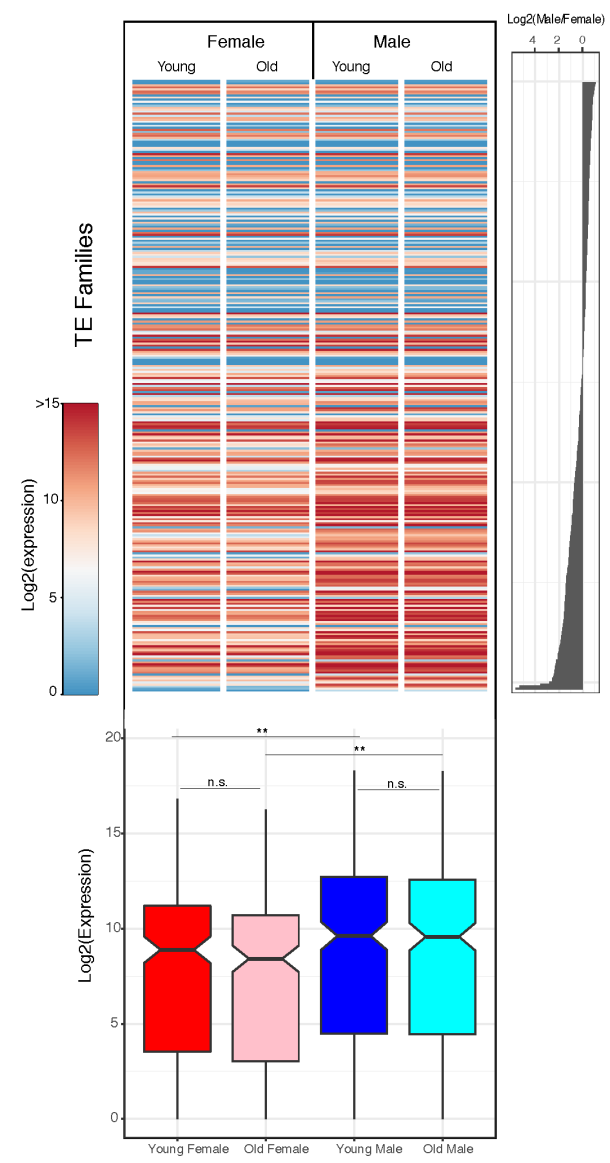

B

C
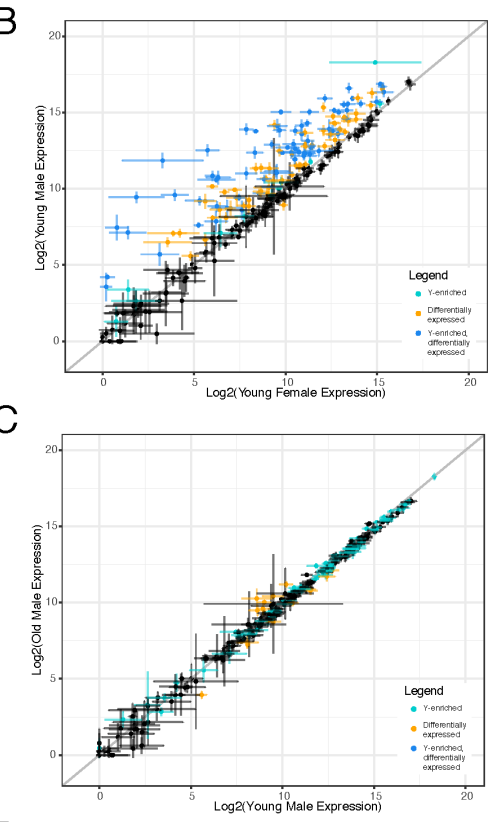
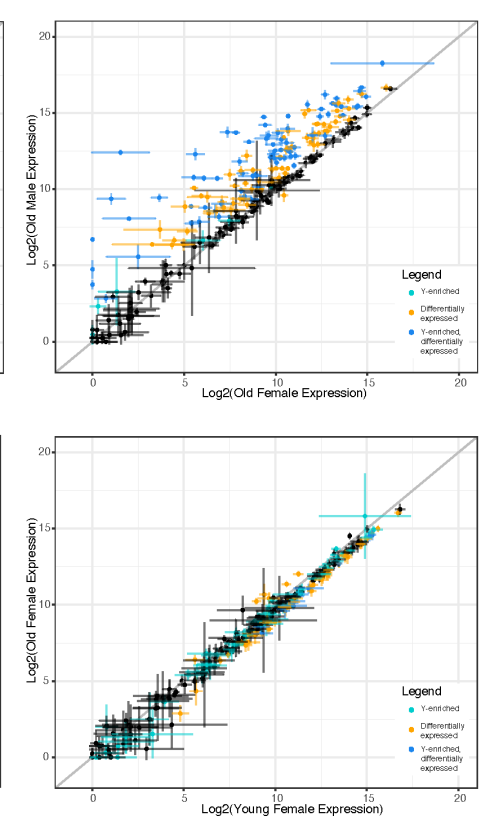

$\mathrm{D}$

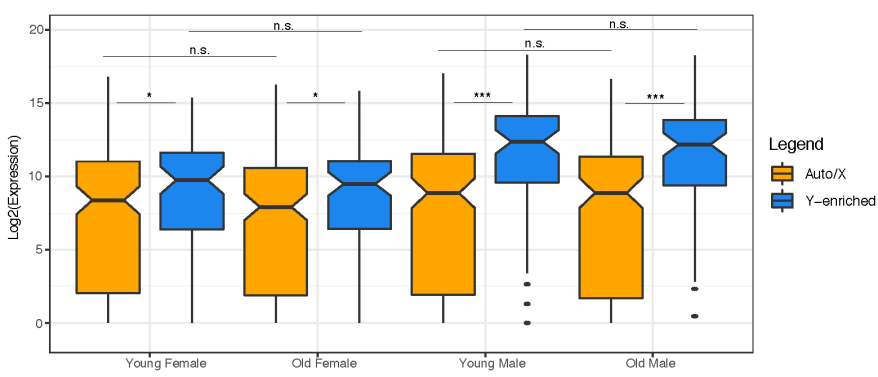

Figure 4. TEs are upregulated in male D. miranda. Shown is differential TE expression within and between sexes. A. Overall log2 expression by sex and age with rows sorted by log2 female/male TE coverage (left histogram). Boxplots below with significance values calculated using the Wilcoxon test $(* \mathrm{p}<0.05, * * \mathrm{p}<0.01, * * * \mathrm{p}<1 \mathrm{e}-5)$. Data represents averaged expression across samples. B. Log2 expression differences of TEs between young (left) and old (right) males versus females. Data indicates mean value of 3 biological replicates and bars denote standard error. Color-code indicates significant comparisons (50\% significant higher or lower expression; Wald test, $p<0.05$ ) and $Y$-enriched repeats (based on genomic coverage): cyan: Y-enriched, $p<0.05$; yellow: unbiased, $p<0.05$; blue: Y-enriched, n.s.; black: unbiased; n.s. Grey line indicates 1:1 expression levels. C. Same as B but comparison between young vs. old males (left) and females (right). D. Log2 TE expression by sex and age grouped by TEs enriched on the neo-Y (blue) and TEs not enriched on the neo-Y (yellow). Significance values calculated using the Wilcoxon test $\left(* p<0.05, * * p<0.01,{ }^{* * *} p<1 e-5\right)$.

It is difficult to identify the genomic copy from which a TE transcript originated, especially for recently active families with highly similar copies. We used several approaches to test if increased TE expression in males is primarily driven by repeats on the neo-Y. First, we mapped RNA seq reads to the $D$. miranda 
genome, and estimated expression of neo-Y linked copies versus copies on other chromosomes for each TE family [33]. We find that $53 \%$ of all TE transcripts in young males are derived from the autosomes and $X$ and $47 \%$ are from the neo- $Y$, suggesting that most of the access TE transcription in males could be attributed to expression from the neo-Y chromosome.

We also quantify expression of TEs enriched on the neo-Y independent of our assembly (Figure 4A,B). TE families with more copies on the neo-Y chromosome will show higher coverage in male DNA sequencing libraries compared to female libraries [12]. We ranked TE families based on their male/female genomic coverage and classified 81 TEs as Y-enriched (based on log2(female/male gDNA) coverage; Wei et al. 2020). Indeed, TE families that are enriched on the neo-Y show higher expression in both males and females than the remaining TE families that are not enriched on the neo-Y (Figure 4D). In addition, most of the neo- $Y$ enriched TE families are significantly more expressed in males, with $85 \%$ of the neo- $Y$ enriched TEs (69 families) being more highly expressed in young males compared to females. Thus, this confirms the expectation that it is more recently active TEs that invaded the neo-Y chromosome since it stopped recombining with the neo-X 1.5MY ago. Increased copy number of these TEs and lower levels of heterochromatin on the neo-Y might drive their higher expression in males.

\section{No change in TE expression in old flies}

A loss of heterochromatin in old individuals can lead to a re-activation of previously silenced TEs [8-11]. In $D$. melanogaster, we found that TEs are efficiently repressed in young male and female brain tissue [12]. While old females maintained heterochromatin and silencing of repeats, males aged for a similar amount of time showed a dramatic reduction in heterochromatin at pericentric regions and on their $Y$ chromosome. Heterochromatin loss in males was accompanied by de-repression of TEs, and repeats from the $Y$ chromosome were found to be disproportionally up-regulated in old $D$. melanogaster males [12].

Female $D$. miranda efficiently suppress TEs throughout their life, similar to $D$. melanogaster. In females, 4 TEs showed increased and 36 decreased expression during aging (Figure 4A, using abs(log2(foldchange) $=0.58$ ), and the total fraction of transcripts derived from repeats decreases during aging (the fraction of repetitive reads in all RNA-seq reads is on average $3.7 \%$ at 9 days, vs. $2.9 \%$ at 80 days, TableS3). In contrast to repeat de-repression found in old $D$. melanogaster males, however, expression of TEs does not change during aging in male $D$. miranda (Figure 4C, Figure S11). In particular, 6/8 TEs show a in/decrease in expression during aging in D. miranda males (Figure 4C, Figure S11; same foldchange as females), and the total fraction of transcripts derived from repeats slightly decreases during aging (from $8.0 \%$ at 9 days to $7.6 \%$ at 80 days, Table S3). Thus, while old D. miranda males show a drastic loss in heterochromatin at pericentromeres on the $\mathrm{X}$ and autosomes, this does not result in global expression changes at TEs. However, unlike in $D$. melanogaster, overall levels of TE expression are substantially increased in male $D$. miranda relative to females, already in young flies (Figure 4B; Figure S11). Note that increased TE expression in male holds even if we account for higher copy number of repeats in males by normalizing by DNA read counts (Figure S13). Thus, higher repeat expression in males is not simply a product of higher TE copy number in males.

\section{Satellite mis-expression in old male flies}

Another type of repetitive DNA that is targeted by heterochromatin is satellite DNA repeats [31]. Satellites are often transcribed, and satellite transcripts may have important biological roles, including heterochromatin formation or centromere assembly [34-37]. Unlike D. melanogaster, which has large arrays of satellite DNA composed of simple repeats (sometimes several megabases in size), the genomes of $D$. miranda and its close relatives contain many fewer, often shorter satellites $[24,38]$. Of the 780 
A

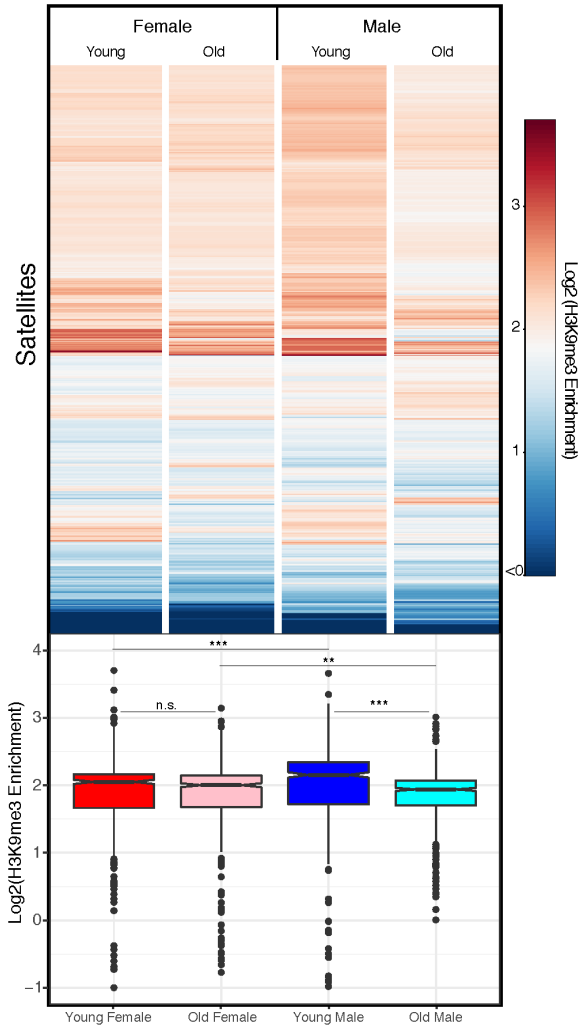

B
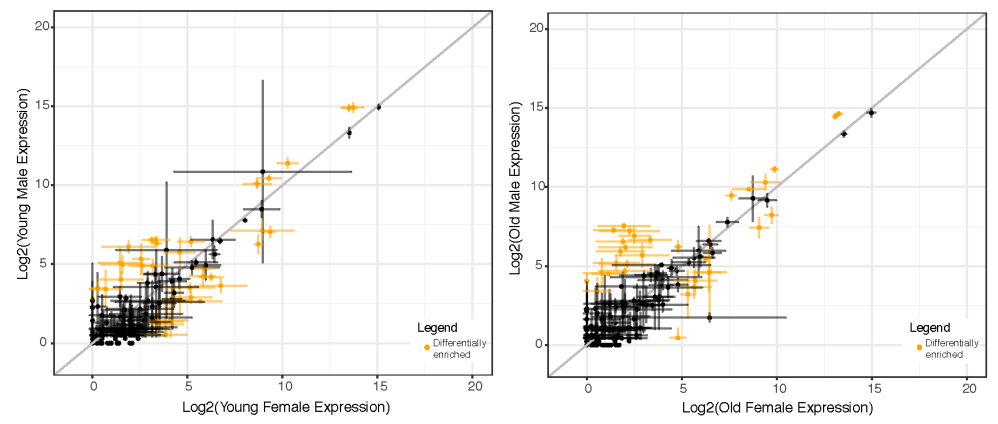

C
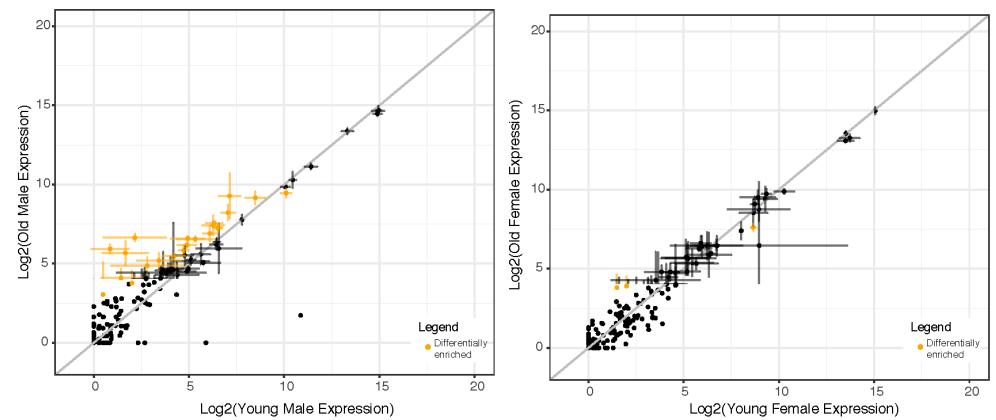

Figure 5. Heterochromatin and expression at satellite DNA. A. Average log2 H3K9me3 enrichment at different satellite DNA for young and old females and males. Boxplots with significance values calculated using the Wilcoxon test $\left({ }^{*} p<0.05, * *\right.$ $\mathrm{p}<0.01, * * * \mathrm{p}<1 \mathrm{e}-5)$. B. Log2 expression differences between young (left) and old (right) males and females. Data represents mean value of 3 replicates with bars denoting standard error, data in yellow denote significant differentially expressed satellites $(50 \%$ higher or lower expression, Wald test, $p<0.05)$. Grey line denotes 1:1 expression levels between the samples. C. Log2 expression differences between young vs. old males (left) and females (right). Plots made in similar fashion as in B.

different satellite repeat units that were identified in the $D$. miranda genome assembly [14], only 9 are enriched on the neo-Y (based on male/female genomic coverage), compared to 81 neo- $Y / Y$ enriched TEs (out of 303 TE families total; see above). Thus, unlike TE's, satellites are not preferentially found on the neo-Y. Satellite DNA is highly enriched for H3K9me3 in young flies (Figure 5A), in both males and females. Mimicking overall patterns of heterochromatin enrichment, we see a clear drop in H3K9me3 levels in old male flies at satellites, but not females (Figure 5A).

Many satellite repeats are transcribed, and at similar levels in both sexes; $21 / 16$ satellites show higher/lower expression in young males compared to young females (Figure 5B, Figure S14). Thus, unlike TEs that are highly enriched on the neo-Y and inefficiently silenced in males (Figure 4), no such differences in satellite expression are seen in young males and females. Old males show a dramatic loss in repressive heterochromatin at satellites, and a simultaneous up-regulation at satellite DNA expression. In males, 21 satellites show increased expression during aging compared to 1 satellite showing decreased expressed, while expression at 2/1 satellites in/decreased in old females (Figure 5C, Figure S14). Thus, a loss of silencing heterochromatin at satellites in old male $D$. miranda is accompanied by a de-repression of satellite transcripts. 


\section{Mis-expression of neo-Y genes and genes embedded in heterochromatin in old flies}

Changes in heterochromatin during aging could also affect protein-coding genes; while most genes are located in repeat-poor euchromatin, some are embedded in heterochromatin. In addition to proteincoding genes located on the neo-Y (5586 in our combined ChIP and RNA analysis), D. miranda also has a total of 376 genes in pericentromeres and the dot. Genes that are naturally located in heterochromatin, such as pericentric genes in Drosophila or genes on the dot chromosome, have evolved mechanisms to assure gene transcription in this normally suppressive chromatin environment, and often are dependent on it [39-41]. Indeed, genes located on the pericentromeres appear to be expressed at slightly lower levels in old flies, compared to young ones, in both sexes (6/18 significantly up/downregulated in aged males and 3/29 in aged females; Figure S15). Neo-Y genes, on the other hand, only became embedded in their heterochromatic environment within the past 1.5MY. Indeed, as flies age and lose H3K9me3, neo-Y genes become slightly upregulated (249/141 are significantly up/downregulated; Figure S15). Thus, these results are consistent with a dynamic view of co-evolution of heterochromatin and its host genome; genes embedded in old heterochromatin, such as the pericentromere, had millions of years of evolution to properly function in this typically repressive environment, and thus depend on it. A loss of heterochromatin during aging thus results in downregulation of pericentric genes. In contrast, genes who only became invaded by repeats and thus heterochromatin more recently, such as neo-Y genes in D. miranda, have not had enough time to adopt to this novel environment; losing heterochromatin, therefore, results in slight upregulation of neo-Y genes.

\section{Discussion}

Repetitive DNA is composed of TEs and satellite DNA, and their misregulation can induce genomic instability [42]. Transposable elements (TEs) are mobile DNA sequences that can change their position and comprise a substantial fraction of eukaryotic genomes [43]. Satellite DNA is often found near centromeres, and incorrect packaging or expression of some satellite DNA can have profound negative fitness consequences [34-37]. Repetitive elements and their host genome are in a constant arms race, which need to co-evolve to evade each other's response $[44,45]$. Mobilization of TEs can cause insertional mutations [43] and natural selection acts to remove deleterious insertions [46]. Several hostencoded mechanisms have emerged during eukaryotic evolution to suppress TE activity [43, 47]. One defense mechanisms widely employed across eukaryotes is transcriptional silencing of repetitive DNA through heterochromatin formation [31]. A host-encoded defense mechanism that prevents movement of a TE family, however, creates selective pressure on these elements to evolve escape mechanisms to avoid the host defense, forcing the host to evolve further mechanisms to silence TEs. Thus, repetitive elements and their host genome are in a constant arms race, where they need to co-evolve to evade each other's response $[44,45]$.

The effectiveness of host defense mechanisms to eliminate or silence repetitive elements can vary in space and time. Epigenetic defense mechanisms can vary both on evolutionary time scales, and throughout an individual's life. Significant chromatin structural changes occur during aging, with a loss of heterochromatin commonly observed in old individuals [5, 29]. Disruption of heterochromatin during an individual's life can result in mobilization of previously silenced TEs $[9,48]$. The effectiveness of epigenetic silencing mechanisms can also vary across evolutionary time scales. In particular, a novel TE that is invading a naïve host genome can escape host surveillance mechanisms and propagate across the genome, until silencing mechanisms evolve to target that TE $[32,49]$. 
Natural selection to remove TE insertions can also be compromised. In the absence of recombination, natural selection is less efficient, and can result in fixation of deleterious mutations, including the accumulation of TEs [50,51]. Low or non-recombining regions of eukaryotic genomes such as pericentromeres are often an agglomerate of TEs $[24,46,52]$ and $Y$ chromosomes of many species, including Drosophila, are comprised almost entirely of repetitive DNA $[52,53]$. In fact, the neo-Y of $D$. miranda has accumulated tens of thousands of TE insertions since it stopped recombining 1.5MY ago $[14,21]$, demonstrating just how quickly TEs can invade a non-recombining chromosome. Nonrecombining $Y$ chromosomes of many species can thus act as reservoirs for TEs [53, 54], and dramatically increase the repeat content in males compared to females [12].

The accumulation of deleterious mutations and repetitive elements on the $Y$ chromosome might lower the survival of the heterogametic sex ("toxic $Y$ " hypothesis) $[3,4]$. While TEs may be efficiently silenced in young individuals, deteriorating heterochromatin can affect males disproportionally, and result in shorter lifespans in males [12]. Under the toxic Y model, we expect males to show higher levels of TE expression than females, at least during some stages in their life (for example, in old males when heterochromatin is known to be compromised). Secondly, we would also expect that larger $Y$ chromosomes that contain more repetitive elements are more 'toxic' to males than $Y$ chromosomes with fewer or less active repeats.

Our data support both predictions of the toxic $Y$ effect. We show that repeats from the $Y$ are misexpressed in male $D$. miranda. We find that the overall number of TE-derived transcripts is about twice as much in males than it is in females, and that TEs located on the $Y$ are primarily responsible for increased TE transcript abundance in males. Secondly, comparison of our data to a recent analysis in $D$. melanogaster suggests that the larger neo-Y of $D$. miranda is a much bigger burden for males than the ancient $Y$ of $D$. melanogaster [12]. Young $D$. melanogaster males are effectively suppressing their $Y$ linked repeats and epigenetic defense mechanisms are only breaking down in old males [12]. In contrast, TE suppression is compromised even in young $D$. miranda males. The $Y$ chromosome of $D$. melanogaster is substantially smaller than the neo-Y of $D$. miranda and fully heterochromatic $[53,54]$. About $1 / 4$ of the $40 \mathrm{Mb}$ large $Y$ chromosome of $D$. melanogaster is composed of TEs (that is, about 10 $\mathrm{Mb}$ ), while the rest of the $\mathrm{Y}$ mainly consists of satellite DNA [13]. In contrast, almost $75 \mathrm{Mb}$ of $\mathrm{Y} / \mathrm{neo}-\mathrm{Y}$ derived sequence in $D$. miranda is classified as TE-derived. In addition to having substantially more repeats, the neo-Y shows lower levels of heterochromatin enrichment compared to repetitive regions on the $\mathrm{X}$ or autosomes. This can explain why $\mathrm{Y}$-linked repeats are ineffectively silenced throughout the life of a fly. Note, however, that our comparative study does not allow us to directly demonstrate that the neo-Y of $D$. miranda reduces male lifespan. Careful genetic manipulations of the sex chromosomes, as done in D. melanogaster [12], are necessary to link the absence or presence of the neo-Y to changes in lifespan.

Overall, we detect higher levels of expression of TEs in male flies relative to female flies, both for young and old flies. Our analysis suggests that low levels of heterochromatin on the neo-Y may be inefficient to fully suppress repetitive elements on this chromosome even in young males. Genome-wide deterioration of heterochromatin in old males may further decrease TE silencing, but may not substantially contribute to overall TE transcript abundance in old males that already have a large, inefficiently silenced highly repeat-rich neo-Y. Indeed, this resembles our findings in $D$. melanogaster flies with additional $Y$ chromosomes [12]. While wildtype $D$. melanogaster males efficiently suppress their Y-linked repeats when young, flies with additional Y chromosomes (XXY females or XYY males) show inefficient silencing of $Y$-linked repeats already in young flies [12]. These patterns are consistent with a dynamic view of co-evolution between selfish TEs and host defense mechanisms. Flies typically 
can suppress wildtype levels of TE activity when young, but these defense mechanisms may fail as flies age (especially in males which have more repetitive DNA) or when they are being challenged with unusually high numbers of active TEs (extra Y chromosomes in XXY and XYY D. melanogaster, or the recently formed, highly repetitive neo-Y of $D$. miranda).

\section{Materials \& Methods}

Drosophila strains. We used the reference genome strain $\mathrm{MSH} 22$ for analysis [14]. For chromatin and gene expression analyses, flies were grown in incubators at $18^{\circ} \mathrm{C}, 60 \%$ relative humidity, and $12 \mathrm{~h}$ light for the indicated number of days following eclosion, and were then flash-frozen in dry ice and stored at $80^{\circ} \mathrm{C}$. Flies were reared on standard molasses fly food: $0.68 \%$ agar $(\mathrm{w} / \mathrm{v}), 2.7 \%$ yeast $(\mathrm{w} / \mathrm{v}), 6.67 \%$ cornmeal (w/v), $0.456 \%$ propionic acid $(\mathrm{v} / \mathrm{v}), 1.6 \%$ sucrose $(\mathrm{w} / \mathrm{v}), 0.76 \%$ of $95 \%$ ethanol $(\mathrm{v} / \mathrm{v}), 0.09 \%$ Tegosept (w/v), 8.2\% molasses (v/v), 0.0625\% CaCl2 (w/v), 0.75\% Na Tartrate (w/v).

Lifespan assays and tissue preparation. Lifespan assays were conducted following \{Linford, 2013 \#2008\} using the same rearing conditions as described above. Briefly, we collected synchronized embryos on a molasses plate with yeast paste for 16-20 hours. We washed embryos with $1 \times$ PBS pH 7.4 three times and dispensed $10 \mu \mathrm{l}$ of embryos per culture vial by pipette. To obtain synchronized adult flies, we collected emerging adults over a 3 day window and aged the adults for at least 7 days to mature and copulate. Afterwards, we separated males and females into separate vials, placing 20 individuals per vial, maintaining them at $18^{\circ} \mathrm{C}$ with a light:dark setting of $12 \mathrm{~h}: 12 \mathrm{~h}$. We moved flies to new vials every $3-5$ days, without using $\mathrm{CO}_{2}$, and fly deaths were recorded. Flies that were observed escaping the vial were censored. To collect samples for the RNA-seq and ChIP-seq experiment, we censored the entire lifespan experiment once it reached less than $50 \%$ survivorship for one sex and flash-froze the remaining flies in liquid nitrogen. In total, 1765 female flies in 107 vials and 1304 male flies in 75 vials were counted for the lifespan assays reported here. We dissected brains from males and females aged 5-9 days (young flies) and 80-98 days old in 1X PBS pH 7.4 (1 brain/sample). We then snap-froze and stored the tissue at $80^{\circ} \mathrm{C}$ prior to use in the ChIP or RNA-seq assay. For the spike-in, we dissected brains from $D$. melanogaster $y w$ strain females and pooled 2 brains/sample.

Chromatin Immunoprecipitation and Sequencing. We modified the native and ultra-low input ChIP protocol [55] for single-brain ChIP-Seq. First, we added $40 \mu \mathrm{L}$ EZ nuclei lysis buffer to frozen tissues and homogenized them with a pestle grinder on ice. We spun cells down at $1000 \mathrm{xg}$ for approximately 10 minutes and decanted $20 \mu \mathrm{L}$ of the supernatant. We froze the cell pellet in the lysis buffer at $-80^{\circ} \mathrm{C}$. We then followed the 100,000 cell count digestion protocol from [55] with a few modifications. For each aged $D$. miranda sample and D. melanogaster spike-in sample, we added $4.4 \mu \mathrm{L}$ of $1 \% \mathrm{DOC}$ and $1 \%$ Triton-X100 to resuspended cells and mixed thoroughly. Briefly, we digested each sample at $37 \mathrm{C}$ for 04:58 (mm:ss) with MNase and quenched the reaction with $4.9 \mu \mathrm{L}$ of $100 \mathrm{mM}$ EDTA pH 8.0. We then added the fragmented spike-in samples to each $D$. miranda sample to account for approximately $20 \%$ of the final pooled sample volume (i.e. final sample consisted of $80 \%$ D. miranda, $20 \%$ D. melanogaster). We reserved $10 \%$ of the pooled fragmented sample for the input (fragment control) and used the remaining $90 \%$ to perform the chromatin pull-down (ChIP sample, target antibody: H3K9me3 polyclonal classic Diagenode C15410056) according to the 100,000 cell specifications. We used the Rubicon Genomics ThruPlex kit to prepare ChIP-Seq libraries for sequencing with 10 PCR amplification cycles for the input samples and 12 cycles for the ChIP pull-down samples. We sequenced libraries on the Illumina HiSeq 4000 Platform at the Vincent J. Coates Genomics Sequencing Center (Berkeley, CA). In total, we 
made 4 biological replicates: 2 replicates from the first aging trial, 1 replicate from the second, and 1 replicate from the third.

RNA extraction and RNA-seq. We extracted total RNA from 20 brains per sex per age ( 3 biological replicas, one from each aging trial) following a standard TRIzol protocol. Briefly, we homogenized tissues first in $100 \mu \mathrm{l} \mathrm{TRIzol}$ and then added $900 \mu \mathrm{I}$ TRIzol to proceed with the full-volume protocol. We generated libraries from total RNA using Illumina's TruSeq Stranded Total RNA Library Prep kit with Ribo-Zero ribosomal RNA reduction chemistry, which depletes the highly abundant ribosomal RNA transcripts (Illumina RS-122-2201). Libraries were run on a Bioanalyzer for fragment traces and sequenced on the Illumina HiSeq 4000 at the Vincent J Coates Genomics Sequencing Center.

Mapping of sequencing reads and data normalization. We mapped all ChIP-seq datasets to the $D$. miranda genome assembly [14] to the $D$. melanogaster Release 6 of the genome assembly and annotation [56]. For all ChIP-seq datasets, we used Bowtie2 [57] to map reads to both genomes separately, using the parameters "-D $15-\mathrm{R} 2-\mathrm{N} 0-\mathrm{L} 22-\mathrm{i}$ S,1,0.50 --no-1-mm-upfront", which allowed us to reduce cross-mapping to the $D$. melanogaster genome to approximately $1 \%$ of $100 \mathrm{bp}$-paired end reads [58]. All ChIP and input samples were processed with bedtools coverage -counts [59]. To calculate the ChIP signal without spike-in normalization, we normalized all counts files by library size, and calculated the coverage across $5 \mathrm{~kb}$ windows for both the ChIP and the input $D$. miranda alignments. These values were then normalized by the median autosome coverage of euchromatic arms (Muller $B$ and Muller E) to obtain ChIP signal (ChIP/Input) for each sample. To calculate signals normalized by the D. melanogaster spike-in, we used three different approaches (see Fig. S1). First, following the approach from [58], we took the ChIP enrichment from the no-spike normalization method and multiplied these values by a scale factor. The scale factor $c$ is calculated as follows with normal script indicating the number of reads mapped for the given sequencing sample and subscripts indicating the specific species to which reads were mapped:

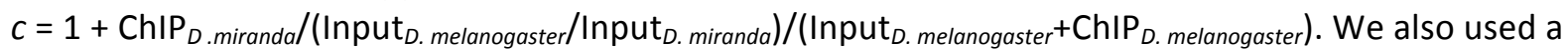
regression-based method from [60]. Briefly, we calculated the median ChIP signal for each sample's $D$. melanogaster spike-ins. Then, the ChIP signal in each $D$. miranda sample was adjusted by a logistic regression based on its $D$. melanogaster spike-in signal. In a separate spike-in normalization approach, we used a quantile-quantile method [61]. A reference ChIP signal for all $D$. melanogaster spike-ins was made by aggregating all $D$. melanogaster ChIP and input coverages, respectively. Then, all individual $D$. melanogaster spike-ins were adjusted to this reference and the corresponding $D$. miranda ChIP signals were adjusted accordingly. All three spike-in normalization methods as well as the no-spike normalization procedure yielded quantitatively similar results: young males and females show similar enrichment of H3K9me3 along their pericentromeres; both old males and females show less H3K9me3 enrichment than young flies, and the loss of heterochromatin is more pronounced in males than females, and finally, the neo-Y chromosome generally shows less heterochromatin than pericentromeres (Fig. S1). We used the no-spike normalization procedure for our analysis, and one sample with low ChIP efficacy (old male 1; see Fig. S1) was excluded from the analysis. The boundaries of euchromatic chromosome arms versus pericentromeric heterochromatin was defined using repeat-enrichment, using a cut-off of $40 \%$ repeat-masked DNA across $100 \mathrm{~kb}$ sliding windows away from the centromere (from [62]. For the neo-Y chromosome, we identified the pericentromeric region based on increased $\mathrm{H} 3 \mathrm{~K} 9 \mathrm{me} 3$ signal surrounding the centromeric satellite sequences (see [14]), which roughly corresponds to $9.8 \mathrm{Mb}$ of sequences at the end of neo-Y scaffold 1.

Gene expression analysis. For each replicate of RNA-seq data, we first mapped RNA-seq reads to a repository of ribosomal DNA scaffolds from NCBI and removed all reads that mapped to this scaffold. 
Although total RNA library preparation aims to remove the bulk of rRNA transcript, any additional differences in rRNA abundance in sequenced samples are likely to be technical artifacts. We then mapped the remaining reads to the $D$. miranda genome and to the repeat libraries separately using HISAT2 [63] with default parameters. We then used Subread FeatureCounts [64] to calculate gene counts and repeat counts and used DESeq2 [65] to normalize the libraries and perform differential expression analysis between male/female flies of different ages. In an analogous approach, we used TECount from TEtranscripts [33] to estimate gene and TE expression counts across samples. We used TECounts with default parameters on RNA-seq reads mapped to the $D$. miranda genome. We also used DESeq2 [60] to perform the differential expression analysis with these counts tables. Both counting methods for repeats yielded similar results for each sample.

Repeat libraries. We used two repeat libraries for the ChIP-seq and RNA-seq analyses. Our first library was the consensus sequences of known repetitive elements identified in the $D$. pseudoobscura group [66] and the second was based on the consensus sequences of known satellites identified in $D$. miranda [14]. The following methods were employed for both repeat libraries.

ChIP enrichment/RNA expression of specific repeats (and by chromosome). To assess H3K9me2 signal in repetitive elements, we took a similar approach as we did for calculating ChIP enrichment profiles across the genome. First, we mapped both ChIP and input sequencing reads to the $D$. pseudoobscura family TE library/satellite DNA library using Bowtie2 [57] and the parameters "-D $15-\mathrm{R} 2-\mathrm{N} 0-\mathrm{L} 22$-i $\mathrm{S}, 1,0.50$--no-1-mm-upfront". We then calculated the mean coverage across each repetitive element using Bedtools [59] coverage, and normalized the coverage by the median autosome coverage. We then calculated the ChIP signal at each repeat by dividing the ChIP coverage by the corresponding input coverage. This method accounts for differences in copy number of the repetitive elements by dividing the ChIP coverage by each repeat's coverage in the input. In a complementary approach, we took ChIP mappings to the entire genome and called enrichment at repetitive elements through TECounts (part of TETranscripts) [33]. To call for repeat enrichment on a per chromosome basis, we split mappings by chromosome and ran TECounts separately for each split mapping. We then normalized counts at repeats in both the ChIP and input by mean autosomal coverage and calculated ChIP enrichment as ChIP/Input.

\section{References}

1. Tarka M, Guenther A, Niemelä PT, et al (2018) Sex differences in life history, behavior, and physiology along a slow-fast continuum: a meta-analysis. Behav Ecol Sociobiol (Print) 72:132-13. doi: 10.1007/s00265-018-2534-2

2. Maklakov AA, Lummaa V (2013) Evolution of sex differences in lifespan and aging: causes and constraints. Bioessays 35:717-724. doi: 10.1002/bies.201300021

3. Xirocostas ZA, Everingham SE, Moles AT (2020) The sex with the reduced sex chromosome dies earlier: a comparison across the tree of life. Biol Lett 16:20190867. doi: 10.1098/rsbl.2019.0867

4. Pipoly I, Bókony V, Kirkpatrick M, et al (2015) The genetic sex-determination system predicts adult sex ratios in tetrapods. Nature 527:91-94. doi: 10.1038/nature15380

5. Larson K, Yan S-J, Tsurumi A, et al (2012) Heterochromatin formation promotes longevity and represses ribosomal RNA synthesis. PLoS Genet 8:e1002473. doi: 10.1371/journal.pgen.1002473

6. Haithcock E, Dayani Y, Neufeld E, et al (2005) Age-related changes of nuclear architecture in Caenorhabditis elegans. Proc Natl Acad Sci USA 102:16690-16695. doi: 10.1073/pnas.0506955102

7. Marais GAB, Gaillard J-M, Vieira C, et al (2018) Sex gap in aging and longevity: can sex chromosomes play a role? Biol Sex Differ 9:33-14. doi: 10.1186/s13293-018-0181-y

8. Wood JG, Jones BC, Jiang N, et al (2016) Chromatin-modifying genetic interventions suppress ageassociated transposable element activation and extend life span in Drosophila. Proc Natl Acad Sci USA 113:11277-11282. doi: 10.1073/pnas.1604621113

9. Wood JG, Hillenmeyer S, Lawrence C, et al (2010) Chromatin remodeling in the aging genome of 
Drosophila. Aging Cell 9:971-978. doi: 10.1111/j.1474-9726.2010.00624.x

10. De Cecco M, Criscione SW, Peckham EJ, et al (2013) Genomes of replicatively senescent cells undergo global epigenetic changes leading to gene silencing and activation of transposable elements. Aging Cell 12:247-256. doi: 10.1111/acel.12047

11. Li W, Prazak L, Chatterjee N, et al (2013) Activation of transposable elements during aging and neuronal decline in Drosophila. Nat Neurosci 16:529-531. doi: 10.1038/nn.3368

12. Brown EJ, Nguyen AH, Bachtrog D (2020) The Y chromosome may contribute to sex-specific ageing in Drosophila. Nat Ecol Evol 37:466-10. doi: 10.1038/s41559-020-1179-5

13. Chang C-H, Larracuente AM (2019) Heterochromatin-Enriched Assemblies Reveal the Sequence and Organization of the Drosophila melanogaster Y Chromosome. Genetics 211:333-348. doi: 10.1534/genetics.118.301765

14. Mahajan S, Wei KH-C, Nalley MJ, et al (2018) De novo assembly of a young Drosophila Y chromosome using single-molecule sequencing and chromatin conformation capture. PLoS Biol 16:e2006348. doi: 10.1371/journal.pbio.2006348

15. Steinemann M, Steinemann S (1992) Degenerating Y chromosome of Drosophila miranda: a trap for retrotransposons. Proc Natl Acad Sci USA 89:7591-7595.

16. Bachtrog D (2006) Expression profile of a degenerating neo-y chromosome in Drosophila. Curr Biol 16:1694-1699. doi: 10.1016/j.cub.2006.07.053

17. Bachtrog D, Hom E, Wong KM, et al (2008) Genomic degradation of a young Y chromosome in Drosophila miranda. Genome Biol 9:R30. doi: 10.1186/gb-2008-9-2-r30

18. Zhou Q, Bachtrog D (2012) Sex-specific adaptation drives early sex chromosome evolution in Drosophila. Science 337:341-345. doi: 10.1126/science.1225385

19. Ellison CE, Bachtrog D (2013) Dosage compensation via transposable element mediated rewiring of a regulatory network. Science 342:846-850. doi: 10.1126/science.1239552

20. Bachtrog D, Charlesworth B (2002) Reduced adaptation of a non-recombining neo-Y chromosome. Nature 416:323-326. doi: 10.1038/416323a

21. Bachtrog D (2003) Accumulation of Spock and Worf, two novel non-LTR retrotransposons, on the neo-Y chromosome of Drosophila miranda. Mol Biol Evol 20:173-181. doi: 10.1093/molbev/msg035

22. Bachtrog D, Mahajan S, Bracewell R (2019) Massive gene amplification on a recently formed Drosophila Y chromosome. Nat Ecol Evol 3:1587-1597. doi: 10.1038/s41559-019-1009-9

23. Zhou Q, Ellison CE, Kaiser VB, et al (2013) The epigenome of evolving Drosophila neo-sex chromosomes: dosage compensation and heterochromatin formation. PLoS Biol 11:e1001711. doi: 10.1371/journal.pbio.1001711

24. Bracewell R, Chatla K, Nalley MJ, Bachtrog D (2019) Dynamic turnover of centromeres drives karyotype evolution in Drosophila. Elife 8:923. doi: 10.7554/eLife.49002

25. Yoon JS, Gagen KP, Zhu DL (1990) Longevity of 68 species of Drosophila. Ohio J Sci 90:16-32.

26. Tower J, Arbeitman M (2009) The genetics of gender and life span. J Biol 8:38-3. doi: 10.1186/jbiol141

27. Brenman-Suttner DB, Yost RT, Frame AK, et al (2020) Social behavior and aging: A fly model. Genes Brain Behav 19:e12598. doi: 10.1111/gbb.12598

28. Li X-Y, Harrison MM, Villalta JE, et al (2014) Establishment of regions of genomic activity during the Drosophila maternal to zygotic transition. Elife 3:e1003428. doi: 10.7554/eLife.03737

29. Sun L, Yu R, Dang W (2018) Chromatin Architectural Changes during Cellular Senescence and Aging. Genes (Basel) 9:211. doi: 10.3390/genes9040211

30. Elgin SCR, Reuter G (2013) Position-effect variegation, heterochromatin formation, and gene silencing in Drosophila. Cold Spring Harb Perspect Biol 5:a017780-a017780. doi: 10.1101/cshperspect.a017780

31. Allshire RC, Madhani HD (2018) Ten principles of heterochromatin formation and function. Nat Rev Mol Cell Biol 19:229-244. doi: 10.1038/nrm.2017.119

32. Molaro A, Malik HS (2016) Hide and seek: how chromatin-based pathways silence retroelements in the mammalian germline. Curr Opin Genet Dev 37:51-58. doi: 10.1016/j.gde.2015.12.001

33. Jin Y, Tam OH, Paniagua E, Hammell M (2015) TEtranscripts: a package for including transposable elements in differential expression analysis of RNA-seq datasets. Bioinformatics 31:3593-3599. doi: 10.1093/bioinformatics/btv422

34. Kuhn GCS (2015) 'Satellite DNA transcripts have diverse biological roles in Drosophila'. Heredity 
(Edinb) 115:1-2. doi: 10.1038/hdy.2015.12

35. Usakin L, Abad J, Vagin VV, et al (2007) Transcription of the 1.688 satellite DNA family is under the control of RNA interference machinery in Drosophila melanogaster ovaries. Genetics 176:1343-1349. doi: $10.1534 /$ genetics.107.071720

36. Topp CN, Zhong CX, Dawe RK (2004) Centromere-encoded RNAs are integral components of the maize kinetochore. Proc Natl Acad Sci USA 101:15986-15991. doi: 10.1073/pnas.0407154101

37. Mills WK, Lee YCG, Kochendoerfer AM, et al (2019) RNA from a simple-tandem repeat is required for sperm maturation and male fertility in Drosophila melanogaster. Elife 8:229. doi: 10.7554/eLife.48940

38. Wei KH-C, Lower SE, Caldas IV, et al (2018) Variable Rates of Simple Satellite Gains across the Drosophila Phylogeny. Mol Biol Evol 35:925-941. doi: 10.1093/molbev/msy005

39. Riddle NC, Shaffer CD, Elgin SCR (2009) A lot about a little dot - lessons learned from Drosophila melanogaster chromosome 4. Biochem Cell Biol 87:229-241. doi: 10.1139/008-119

40. Yasuhara JC, DeCrease CH, Wakimoto BT (2005) Evolution of heterochromatic genes of Drosophila. Proc Natl Acad Sci USA 102:10958-10963. doi: 10.1073/pnas.0503424102

41. Yasuhara JC, Wakimoto BT (2008) Molecular landscape of modified histones in Drosophila heterochromatic genes and euchromatin-heterochromatin transition zones. PLoS Genet 4:e16. doi: 10.1371/journal.pgen.0040016

42. Ivics Z, Izsvák Z (2010) Repetitive elements and genome instability. Semin Cancer Biol 20:197-199. doi: 10.1016/j.semcancer.2010.08.002

43. Bourque G, Burns KH, Gehring M, et al (2018) Ten things you should know about transposable elements. Genome Biol 19:199. doi: 10.1186/s13059-018-1577-z

44. Werren JH (2011) Selfish genetic elements, genetic conflict, and evolutionary innovation. Proc Natl Acad Sci USA 108 Suppl 2:10863-10870. doi: 10.1073/pnas.1102343108

45. Hurst GD, Werren JH (2001) The role of selfish genetic elements in eukaryotic evolution. Nat Rev Genet 2:597-606. doi: 10.1038/35084545

46. Charlesworth B, Langley CH (1986) The evolution of self-regulated transposition of transposable elements. Genetics 112:359-383.

47. Cosby RL, Chang N-C, Feschotte C (2019) Host-transposon interactions: conflict, cooperation, and cooption. Genes Dev 33:1098-1116. doi: 10.1101/gad.327312.119

48. O'Sullivan RJ, Karlseder J (2012) The great unravelling: chromatin as a modulator of the aging process. Trends Biochem Sci 37:466-476. doi: 10.1016/j.tibs.2012.08.001

49. Bousios A, Nützmann H-W, Buck D, Michieletto D (2020) Integrating transposable elements in the 3D genome. Mob DNA 11:8-10. doi: 10.1186/s13100-020-0202-3

50. Charlesworth B, Charlesworth D (2000) The degeneration of Y chromosomes. Philos Trans R Soc Lond, B, Biol Sci 355:1563-1572. doi: 10.1098/rstb.2000.0717

51. Hill WG, Robertson A (1966) The effect of linkage on limits to artificial selection. Genet Res 8:269294.

52. Bachtrog D (2013) Y-chromosome evolution: emerging insights into processes of Y-chromosome degeneration. Nat Rev Genet 14:113-124. doi: 10.1038/nrg3366

53. Pimpinelli S, Berloco M, Fanti L, et al (1995) Transposable elements are stable structural components of Drosophila melanogaster heterochromatin. Proc Natl Acad Sci USA 92:3804-3808.

54. Gatti M, Pimpinelli S (1992) Functional elements in Drosophila melanogaster heterochromatin. Annu Rev Genet 26:239-275. doi: 10.1146/annurev.ge.26.120192.001323

55. Brind'Amour J, Liu S, Hudson M, et al (2015) An ultra-low-input native ChIP-seq protocol for genomewide profiling of rare cell populations. Nat Commun 6:6033. doi: 10.1038/ncomms7033

56. Hoskins RA, Carlson JW, Wan KH, et al (2015) The Release 6 reference sequence of the Drosophila melanogaster genome. Genome Res 25:445-458. doi: 10.1101/gr.185579.114

57. Langmead B, Salzberg SL (2012) Fast gapped-read alignment with Bowtie 2. Nat Methods 9:357-359. doi: 10.1038/nmeth.1923

58. Brown EJ, Nguyen AH, Bachtrog D (2020) The Drosophila Y chromosome affects heterochromatin integrity genome-wide. Mol. Biol. Evol.

59. Quinlan AR, Hall IM (2010) BEDTools: a flexible suite of utilities for comparing genomic features. Bioinformatics 26:841-842. doi: 10.1093/bioinformatics/btq033

60. Bonhoure N, Bounova G, Bernasconi D, et al (2014) Quantifying ChIP-seq data: a spiking method 
providing an internal reference for sample-to-sample normalization. Genome Res 24:1157-1168. doi: 10.1101/gr.168260.113

61. Wei K HC, Gibilisco L, Bachtrog D, (2020) Epigenetic conflict on a degenerating Y chromosome increases mutational burden in Drosophila males.

bioRxiv 2020.07.19.210948; doi: https://doi.org/10.1101/2020.07.19.210948

62. Bracewell R, Chatla K, Nalley MJ, Bachtrog D (2019) Dynamic turnover of centromeres drives karyotype evolution in Drosophila. Elife 8:923. doi: 10.7554/eLife.49002

63. Kim D, Langmead B, Salzberg SL (2015) HISAT: a fast spliced aligner with low memory requirements. Nat Methods 12:357-360. doi: 10.1038/nmeth.3317

64. Liao Y, Smyth GK, Shi W (2014) featureCounts: an efficient general purpose program for assigning sequence reads to genomic features. Bioinformatics 30:923-930. doi: 10.1093/bioinformatics/btt656

65. Love MI, Huber W, Anders S (2014) Moderated estimation of fold change and dispersion for RNA-seq data with DESeq2. Genome Biol 15:550-21. doi: 10.1186/s13059-014-0550-8

66. Hill T, Betancourt AJ (2018) Extensive exchange of transposable elements in the Drosophila pseudoobscura group. Mob DNA 9:20. doi: 10.1186/s13100-018-0123-6

67. Kaplan EL, Meier P (1958) Nonparametric Estimation from Incomplete Observations. Journal of the American Statistical Association 53:457-481. doi: 10.1080/01621459.1958.10501452

\section{Data availability}

All the sequencing data have been posted on GenBank under BioProject PRJNA644734. All processed data files have been posted on Dryad.

\section{Acknowledgments}

This work was supported by NIH grants (nos. R01GM076007, R01GM101255 and R01AG057029) to DB.

Author contributions. AHN collected and analyzed the data, DB wrote the paper with input from AHN. The authors declare no competing financial interests. Correspondence and requests for materials should be addressed to dbachtrog@berkeley.edu. 